\title{
Spécificité de champignons ectomycorhiziens vis-à-vis d'Eucalyptus viminalis Labill et $E$ dunnii Maiden
}

\author{
VL de Oliveira*, VDB Schmidt, NC Gomes, DC Maia \\ Dept de Microbiologia e Parasitologia, Universidade Federal de Santa Catarina, CP 476, \\ 88040-900 Florianópolis-SC, Brésil
}

(Reçu le 9 avril 1993; accepté le 2 décembre 1993)

\begin{abstract}
Résumé - La capacité infectieuse de 19 souches ectomycorhiziennes a été étudiée en serre vis-à-vis d'Eucalyptus dunnii et $E$ viminalis. L'inoculum, produit dans un mélange vermiculite-tourbe $(4: 1, \mathrm{v} / \mathrm{v})$ humidifié avec le milieu MNM et incubé à $25^{\circ} \mathrm{C}$ pendant $45 \mathrm{j}$, a été ajouté $(1: 10, \mathrm{v} / \mathrm{v})$ au substrat de croissance des plants (sable-vermiculite-tourbe, 50:45:5, v/v/v). Ce mélange a été distribué dans les conteneurs et semé avec 1 des 2 espèces d'eucalyptus. Les plants ont été maintenus en serre $\left(25^{\circ} \mathrm{C} \pm 5^{\circ} \mathrm{C}, 16 \mathrm{~h}\right.$ de photopériode) pendant $101 \mathrm{j}$. Après cette période, les plants ont été prélevés et les racines lavées et séparées de la partie aérienne pour l'observation du taux de colonisation. Trois souches de Pisolithus tinctorius ont été spécifiques d'E dunnii. Aucune des souches n'a présenté de spécificité vis-à-vis d'E viminalis. Une autre souche de cette espèce a montré un taux de colonisation plus important chez $E$ dunnii que chez $E$ viminalis. Deux souches isolées sous Pinus spp ont colonisé les 2 espèces. Ces résultats démontrent que la spécificité de certaines souches ectomycorhiziennes peut être observée même entre des espèces hôtes du même genre et est indépendante de l'origine géographique de la souche. Ces informations sont très utiles pour l'établissement de programmes de contrôle de la mycorhization. Les résultats démontrent que la sélection préalable de la souche est un des facteurs essentiels pour le succès de ces programmes.
\end{abstract}

Eucalyptus dunnii/ Eucalyptus viminalis/champignons ectomycorhiziens / spécificité

Summary - Specificity of ectomycorrhizal fungi towards Eucalyptus viminalis Labill and E dunnii Maiden. The ability of 19 ectomycorrhizal fungal isolates to infect Eucalyptus dunnii and $\mathrm{E}$ viminalis was studied in greenhouse conditions. Fungal inocula were grown for $45 d$ at $25^{\circ} \mathrm{C}$ in a vermiculite-peat mixture $(4: 1, \mathrm{~V} / \mathrm{V})$ moistened with nutrient medium (MMM). The colonized substrate was then added at a rate of 1:10 parts of a mixture of sand-vermiculite-peat $(50: 45: 5, \mathrm{v} / \mathrm{v} / \mathrm{v})$ and distributed in plastics pots $(60 \mathrm{ml}$ per pot) before sowing. Seeded pots were maintained in the greenhouse at $25 \pm 5^{\circ} \mathrm{C}$ and $16 \mathrm{~h}$ of daily photoperiod for $101 d$, after which the seedlings were harvested. The roots were removed from the shoots and gently washed free of substrate. They were then examined with the aid of a stereomicroscope to determine the extent of mycorrhizal development. Of the fungi tested, 3 isolates of Pisolithus tinctorius were specific for $\mathrm{E}$ dunnii but none were specific for $\mathrm{E}$ viminalis. One isolate of $\mathrm{P}$ tinctorius showed significantly greater colonization of $\mathrm{E}$ dunnii than $\mathrm{E}$ viminalis. Two isolates from Pinus spp infected both species. These results indicate that the fungi differ in their compatibility for host species, showing that pre-screening is important to the success of mycorrhization control programmes.

Eucalyptus dunnii / Eucalyptus viminalis / ectomycorrhizal fungi/ specificity

\section{INTRODUCTION}

L'eucalyptus est la principale plante exotique utilisée au Brésil dans les programmes de re- boisement. Chez plusieurs entreprises forestières, les plants d'eucalyptus sont produits en pépinière dans un substrat désinfecté et sont transplantés, généralement, dans des sites hébergeant auparavant des espèces indi-

" Correspondance et tirés à part. 
gènes, dont le status ectomycorhizien est peu connu. Certaines plantations sont parfois établies dans des sites plantés préalablement avec Pinus spp, où l'existence de souches mycorhiziennes compatibles avec les eucalyptus est à déterminer. Des observations comparatives effectuées dans certaines plantations de Pinus et Eucalyptus dans le nord de l'État de Santa Catarina (région sud du Brésil) ont suggéré que les eucalyptus et les pins sont associés à différents types de champignons ectomycorhiziens (de Oliveira et al, 1991). L'absence d'un programme de contrôle de la mycorhization pour les eucalyptus ne permet pas d'assurer leur colonisation sur le terrain.

Bien que la spécificité des champignons ectomycorhiziens soit considérée comme un phénomène peu fréquent (Chilvers, 1973; Harley et Smith, 1983), certaines études ont démontré son existence chez quelques couples champignon-plante hôte (Malajczuk et al, 1982; Molina et Trappe, 1982; Malajczuk et al, 1984; Helsin et Douglas, 1986; Lei et al, 1990; Malajczuk et al, 1990; Thoen et al, 1990). Deux cas de spécificité chez les Eucalyptus ont été observés par Malajczuk et al (1982), où les champignons Hymenogaster albellus et Melanogaster intermedius ont sélectivement colonisé certaines espèces de ce genre. Molina et Trappe (1982) ont aussi ob- servé des cas de spécificité de certaines espèces fongiques vis-à-vis de Pinus spp.

Un programme de contrôle de la mycorhization pour les eucalyptus avant transplantation peut être une très importante pratique pour augmenter la productivité des plantations (Lapeyrie et al, 1992). Dans ces programmes, la détermination préalable de la compatibilité vis-à-vis des espèces végétales, des souches mycorhiziennes à introduire, est indispensable (Malajczuj et al, 1982).

Dans ce cadre, cette étude a été faite pour déterminer la capacité de souches ectomycorhiziennes à infecter les plants d'Eucalyptus dunnii et $E$ viminalis, ceci visant à leur future utilisation dans les programmes de contrôle de la mycorhization dans l'État de Santa Catarina.

\section{MATÉRIEL ET MÉTHODES}

\section{Les plantes hôtes et les souches fongiques}

Dans cette étude, l'infectivité de certaines souches de champignons ectomycorhiziens vis-à-vis d'E dunnii et $E$ viminalis a été étudiée dans 3 expériences, sous serre. Les souches employées ont été choisies parmi les souches disponibles au laboratoire de microbiologie de l'université d'État de Santa Catarina. Les espèces et leurs origines respectives sont présentées dans le tableau 1.

Tableau I. Souches fongiques ectomycorhiziennes utilisées avec leurs plante-hôte et provenances respectives.

\begin{tabular}{|c|c|c|c|}
\hline Souches & Espèces & Plante-hôte & Provenance $^{\mathrm{b}}$ \\
\hline $\mathrm{Pi}-4$ & Paxillus involutus & Eucalyptus dalrympleana & Australie \\
\hline Marx 298 & Pisolithus tinctorius & Pinus taeda & États-Unis \\
\hline NI-12 & Gastéromycète non identifié & Pinus sp & Três Barras-SC-Brésil \\
\hline Pt-21 & $P$ tinctorius & Eucalyptus sp & Três Barras-SC-Brésil \\
\hline $\mathrm{Pt}-23$ & $P$ tinctorius & Eucalyptus sp & Florianópolis-SC-Brésil \\
\hline Pt-24 & $P$ tinctorius & $"$ & Florianópolis-SC-Brésil \\
\hline $\mathrm{Pt}-26$ & $P$ tinctorius & $"$ & Florianópolis-SC-Brésil \\
\hline Pt-27 & $P$ tinctorius & $"$ & Florianópolis-SC-Brésil \\
\hline Pt-29 & $P$ tinctorius & $"$ & Florianópolis-SC-Brésil \\
\hline Sc-42 & Scleroderma sp. & Pinus sp & Blumenau-SC-Brésil \\
\hline Pt-43 & $P$ tinctorius & Eucalyptus sp & Florianópolis-SC-Brésil \\
\hline Pt-44 & $P$ tinctorius & $"$ & Florianópolis-SC-Brésil \\
\hline $\mathrm{H}-4942$ & Gastéromycète non identifié & $"$ & Australie \\
\hline $\mathrm{H}-4943$ & $P$ tinctorius & $"$ & Australie \\
\hline $\mathrm{H}-4937$ & $P$ tinctorius & $"$ & Australie \\
\hline Pt-49 & $P$ tinctorius & $"$ & Florianópolis-SC-Brésil \\
\hline$R V-82$ & $P$ tinctorius & Eucalyptus grandis & Viçosa-MG-Brésil \\
\hline$A C-3$ & $P$ tinctorius & Eucalyptus torelliana & Timóteo-MG-Brésil \\
\hline ITA-6 & $P$ tinctorius & " & Itamarandiba-MG-Brésil \\
\hline
\end{tabular}

${ }^{a}$ Espèce végétale d’où la souche a été isolée. ${ }^{b} \mathrm{SC}$ : État de Santa Catarina (région sud du Brésil); MG : état de Minas Gerais (région sud-est du Brésil). 


\section{Production de l'inoculum fongique}

L'inoculum mycélien a été produit dans un mélange tourbe-vermiculite $(1: 4, v / v)$, enrichi avec le milieu liquide Melin Norkrans modifié (MNM) (Marx, 1969). Ce mélange a été distribué dans des bocaux en verre d'environ $1000 \mathrm{ml}$ de capacité ( $300 \mathrm{ml}$ du mélange $+150 \mathrm{ml}$ de milieu par bocal) et stérilisé à l'autoclave à $120^{\circ} \mathrm{C}$ pendant $20 \mathrm{~min}$. Les couvercles des bocaux étaient équipés d'un dispositif permettant l'aération sans compromettre la stérilité du contenu (Marx et Kenney, 1982).

Les souches ont été préalablement cultivées en milieu MNM gélosé en boîtes de Petri à $25^{\circ} \mathrm{C}$ pendant 3 sem. À partir de ces cultures, des implants de $6 \mathrm{~mm}$ de diamètre ont été prélevés aseptiquement à la périphérie des colonies et utilisés pour ensemencer le mélange en surface, à raison de 12 implants par bocal. Ces cultures ont été ensuite mises en incubation à $25^{\circ} \mathrm{C}$, à l'obscurité.

\section{Inoculation du substrat de production des plants}

Au bout de 45 j d'incubation, quand le champignon a colonisé entièrement le substrat, l'inoculum a été apporté dans un mélange sable-vermiculite-tourbe stérile (50:45:5, v/v/v), utilisé par Thoen et Ducousso (1989), à raison de $1: 10(\mathrm{v} / \mathrm{v})$ et homogénéisé manuellement. Ce substrat a été placé ensuite dans les conteneurs et humidifié à l'eau distillée avant le semis des graines. Les traitements témoins, dans chaque expérience, ont été faits par addition dans le substrat d'un mélange d'inoculum de différentes souches, préalablement stérilisé à l'autoclave à $120^{\circ} \mathrm{C}$ pendant $20 \mathrm{~min}$.

\section{Les conditions expérimentales}

Des pots en polypropylène (système dibble-tube), de $60 \mathrm{ml}$ de capacité, employés couramment pour la production de plants d'eucalyptus par les entreprises forestières de la région, ont été utilisés comme conteneurs.

Chaque pot a reçu alors 10 graines d'une des 2 espèces et l'ensemble a été placé en serre en conditions contrôlées de température $\left(25 \pm 5^{\circ} \mathrm{C}\right)$ et de lumière ( $16 \mathrm{~h}$ de jour). Une sem après le semis, les plants en excès ont été coupés de façon à laisser un plant par pot. Chaque expérience comportait 5 répétitions par traitement, avec un plant par répétition, dans une distribution en randomisation totale.

Les plants ont été arrosés avec de l'eau distillée $5 \mathrm{j}$ par sem, et $2 \mathrm{j}$ par sem avec une solution nutritive légèrement modifiée par rapport à celle mise au point par Garbaye, décrite par Leyval (1988), dont la composition est la suivante, en $\mathrm{mg} / \mathrm{L}$ : $\mathrm{Ca}\left(\mathrm{H}_{2} \mathrm{PO}_{4}\right)_{2} \cdot \mathrm{H}_{2} \mathrm{O}, 43,7 ;\left(\mathrm{NH}_{4}\right)_{2} \mathrm{SO}_{4}, 66,0 ; \mathrm{K}_{2} \mathrm{SO}_{4}$, 22,3; $\mathrm{MgSO}_{4} \cdot 7 \mathrm{H}_{2} \mathrm{O}, 51,2 ; \mathrm{CaCl}_{2} \cdot 2 \mathrm{H}_{2} \mathrm{O}, 66,2$;
$\mathrm{H}_{3} \mathrm{BO}_{3}, 2,3 ; \mathrm{MnSO}_{4} \cdot \mathrm{H}_{2} \mathrm{O}, 1,2 ; \mathrm{ZnSO}_{4} \cdot 7 \mathrm{H}_{2} \mathrm{O}, 2,0$ $\mathrm{CuCl}_{2} \cdot 5 \mathrm{H}_{2} \mathrm{O}, 0,52 ; \mathrm{MoO}_{3}, 0,6$.

La première expérience a été menée pendant une période allant de fin janvier à début mai 1990 , la deuxième entre septembre et décembre 1990 et la troisième entre décembre 1990 et mars 1991.

\section{Observations}

Après $101 \mathrm{j}$ de croissance, les plants ont été extraits des pots et le système racinaire a été séparé de la partie aérienne. Les racines ont été lavées à l'eau courante et stockées au froid $\left( \pm 4^{\circ} \mathrm{C}\right)$ dans une solution de formol à $5 \%$, pendant 2 à $4 \mathrm{j}$, le temps nécessaire pour l'observation de tous les plants.

Pour la détermination du taux de mycorhization, des échantillons d'environ $1 \mathrm{~g}$ de racines de chaque plant ont été découpés en morceaux d'environ $2 \mathrm{~cm}$ et observés à la loupe binoculaire $(30 \times)$ pour déterminer la proportion de racines courtes mycorhizées et non mycorhizées pour un total de 1000 racines courtes observées.

\section{RÉSULTATS}

\section{Expérience 1}

Le tableau II présente les résultats de colonisation mycorhizienne pour les 2 espèces dans la première expérience, au cours de la-

Tableau II. Pourcentage de colonisation d'E dunnii et $E$ viminalis inoculés avec différentes souches fongiques ectomycorhiziennes, après $101 \mathrm{j}$ de croissance $^{\mathrm{a}}$. Expérience 1.

\begin{tabular}{lcc}
\hline \multicolumn{1}{c}{ Traitement } & E dunnii & E viminalis \\
\hline Marx 298 & 25,9 & 29,7 \\
NI-12 & 31,8 & 30,0 \\
Pt-29 & 32,9 & 25,3 \\
H-4943 & 36,0 & 7,8 \\
Pt-49 & 10,2 & 22,3 \\
Pt-26 & 31,5 & 0,0 \\
Pt-44 & 23,4 & 0,0 \\
Pt-23 & 11,9 & 0,0 \\
Pt-27 & 0,0 & 0,0 \\
H-4937 & 0,0 & 0,0 \\
Témoin & 0,0 & 0,0 \\
\hline
\end{tabular}

aLes valeurs représentent la moyenne de 5 plants par traitement. 
quelle ont été testées 10 souches fongiques de différentes origines.

Ces résultats montrent que 5 de ces souches, parmi lesquelles 4 de l'espèce $P i$ solithus tinctorius, ont été capables de coloniser les 2 espèces. Les 5 autres souches de $P$ tinctorius testées ont présenté des résultats variables. Trois d'entre elles ont colonisé seulement les plants d'E dunnii tandis que les 2 autres n'ont infecté aucune des 2 espèces.

D'autre part, une des souches $(\mathrm{H}-4943)$ a présenté une plus forte intensité de colonisation chez $E$ dunnii (36\%) que chez $E$ viminalis $(7,8 \%)$ tandis que la souche Pt-49 a colonisé davantage $E$ viminalis (22,3\%) qu'E dunnii $(10,2 \%)$.

En ce qui concerne le comportement des souches dans chaque espèce hôte, il est possible d'observer que, parmi les 10 souches testées, 7 ont colonisé les plants d'E dunnii et seulement 4 ont colonisé ceux d'E viminalis.

II faut noter que les 2 souches isolées sous Pinus spp, une provenant des États-Unis et l'autre isolée à Santa Catarina, ont colonisé les 2 espèces hôtes testées, avec des taux de colonisation comparables dans les 2 cas.

\section{Expérience 2}

Dans la deuxième expérience, effectuée avec 5 souches, dont les résultats sont présentés sur le tableau III, 3 souches ont infecté les plants d'E dunnii et seulement 2 ont infecté ceux d'E viminalis. Parmi ces souches, la souche $\mathrm{H}-4942$ (Gastéromycète hypogé non identifié, isolé en Australie, Collection CRFINRA, Nancy, France) a colonisé $32,0 \%$ de racines chez $E$ dunnii et $50,6 \%$ chez $E$ viminalis.

Tableau III. Pourcentage de colonisation d'E dunnii et $E$ viminalis incolulés avec différentes souches ectomycorhiziennes, après $101 \mathrm{j}$ de croissance ${ }^{\mathrm{a}}$. Expérience 2.

\begin{tabular}{lcc}
\hline \multicolumn{1}{c}{ Traitement } & E dunnii & E viminalis \\
\hline $\mathrm{H}-4942$ & 32,0 & 50,6 \\
$\mathrm{RV}-82$ & 7,7 & 7,0 \\
$\mathrm{Pi}-4$ & 1,3 & 0,0 \\
$\mathrm{Pt}-23$ & 0,0 & 0,0 \\
$\mathrm{Pt}-27$ & 0,0 & 0,0 \\
Témoin & 0,0 & 0,0 \\
\hline
\end{tabular}

${ }^{a}$ Les valeurs représentent la moyenne de 5 plants par traitement.
Parmi les souches de $P$ tinctorius, la souche RV-82, isolée dans l'État de Minas Gerais, s'est montrée la plus infective avec 7,7 et $7,0 \%$, de colonisation chez $E$ dunnii et $E$ viminalis, respectivement. $\mathrm{La}$ souche $\mathrm{Pi}-4$ a présenté un taux de colonisation très réduit $(1,3 \%)$ et cela seulement pour les plants d'E dunnii.

Les souches Pt-23 et Pt-27, déjà testées dans la première expérience (tableau II), ont encore été employées dans cette deuxième expérience. En ce qui concerne la souche Pt17 , son comportement vis-à-vis des 2 espèces s'est montré comparable à celui de l'expérience précédente. Cette souche n'a pas été capable d'infecter les 2 eucalyptus dans les 2 expériences. En revanche, la souche Pt-23, qui s'est montrée infective vis-à-vis d' $E$ dunnii dans la première, n'a pas présenté de pouvoir infectieux dans cette expérience.

\section{Expérience 3}

Dans la troisième expérience, 6 nouvelles souches fongiques ont été étudiées (tableau IV). Les résultats montrent que la compatibilité des souches est la même pour les 2 espèces. On remarque néanmoins que la souche Pt-24, isolée dans les plantations d'E viminalis, à Três Barras, Santa Catarina, présente une intensité de colonisation supérieure aux autres souches, pour les 2 espèces hôtes.

Dans un autre ordre d'idée, les taux de coIonisation sont plus faibles que dans les expériences précédentes. Tandis que, dans la première expérience, ils ont atteint des valeurs supérieures à $30 \%$, dans celle-ci, les va-

Tableau IV. Pourcentage de colonisation d'E dunnii et $E$ viminalis inoculés avec différentes souches ectomycorhiziennes, après $101 \mathrm{j}$ de croissance ${ }^{a}$. Expérience 3 .

\begin{tabular}{lcc}
\hline \multicolumn{1}{c}{ Traitement } & E dunnii & E viminalis \\
\hline Pt-24 & 12,0 & 14,2 \\
ITA-6 & 4,0 & 4,9 \\
AC-3 & 3,4 & 3,9 \\
Pt-43 & 4,1 & 3,7 \\
Sc-42 & 3,3 & 3,4 \\
Pt-21 & 3,0 & 2,8 \\
Témoin & 0,08 & 0,3 \\
\hline
\end{tabular}

${ }^{a}$ Les valeurs représentent la moyenne de 5 plants par traitement. 
leurs plus importantes ont été de $12 \%$ et $14 \%$, et cela pour une seule souche, Pt-24.

\section{DISCUSSION}

Les résultats des expériences montrent que les souches testées présentent des différences dans leur aptitude à coloniser les 2 espèces, dans les conditions utilisées. Nous avons observé une spécificité entre les souches, notamment dans la première expérience, où les taux de mycorhization ont été plus importants que dans les expériences suivantes.

Certaines souches ont infecté sélectivement les 2 espèces étudiées et cela indépendamment de l'origine des souches. Les souches Pt-23, Pt-26 et Pt-44, toutes les 3 isolées sous Eucalyptus spp ont été infectives seulement pour les plants d'E dunnii. En revanche, les souches $\mathrm{NI}-12$ et Marx 298, isolées sous Pinus spp ont significativement colonisé les 2 espèces. La spécificité est encore observée pour les souches Pt-27, H4937, obtenues dans des plantations d'Eucalyptus spp qui ne sont pas parvenues à coloniser les 2 espèces dans les conditions de ces expériences.

Ces résultats rejoignent les observations faites par Molina et Trappe (1982) pour les champignons Hysterangium separabile et Suillus cavipes par rapport à 3 Pinus spp et par Malajczuk et al (1982) pour Hymenogaster albellus et Melanogaster intermedius chez Eucalyptus spp. L'affirmation de Chilvers (1973), sur l'existence d'une spécificité des champignons ectomycorhiziens au niveau du genre plutôt qu'au niveau de l'espèce hôte, est à revoir.

Toutefois, il est possible que les conditions expérimentales puissent contribuer à l'expression de ces résultats, comme il a été suggéré par Harley et Smith (1973). II est aussi possible que la culture continue des souches en laboratoire puisse être responsable de la perte de pouvoir infectieux de certaines souches. Des cas semblables ont été observés par Marx (1981). Si cette dernière suggestion peut expliquer l'absence d'infection de la part de quelques souches, cela n'explique pas la spécificité observée pour les autres souches.
II est intéressant de noter que les souches de $P$ tinctorius se comportent différemment vis-à-vis des 2 espèces, indépendamment de l'origine géographique des souches. Ce fait indique l'existence de différents degrés de compatibilité champignon-plante hôte dans une même espèce fongique et vient confirmer les résultats de Heslin et Douglas (1986), Malajczuk et al (1990) et Jacobson et Miller (1992).

\section{REMERCIEMENTS}

Cette étude a été financée par la Commission des Communautés européennes [Contrat TS2A-0093 F $(S P)]$. Les auteurs expriment leur gratitude au CNPq (Conselho Nacional de Desenvolvimento Científico e Tecnológico)-RHAE pour les bourses de recherches accordées et à Rigesa, Celulose, Papel e Embalagens Ltda pour toutes les facilités dans l'exécution de ce travail.

\section{RÉFÉRENCES}

Chilvers GA (1973) Host range of some eucalypt mycorrhizal fungi. Aust $J$ Bot 21, 103-111

de Oliveira VL, Schmidt VDB, Bellei MM (1991) Dinâmica das simbioses micorrízicas em Eucalyptus dunnii em Santa Catarina. Programas e Resumos da IV Reunião Brasileira sobre Micorrizas, 140

Harley JL, Smith SE (1983) Mycorrhizal symbiosis. Academic Press, London, $483 p$

Heslin MC, Douglas GC (1986) Synthesis of poplar mycorrhizas. Trans Br Mycol Soc, 86, 117-122

Jacobson KM, Miller OK Jr (1992) Physiological variation between tree-associated populations of Suillus granulatus as determined by in vitro mycorrhizal synthesis. Can J Bot 70, 26-31

Lapeyrie F, Garbaye J, de Oliveira V, Bellei M (1992) Controlled mycorrhization of eucalypts. In: Mycorrhizal in ecosystems. (DJ Read, DH Lewis, AH Fitter, IJ Alexander, eds) CAB International, London, 293-299

Lei J, Lapeyrie F, Malajczuk N, Dexheimer J (1990) Infectivity of isolates of Pisolithus tinctorius (Pers) Coker and Couch on roots of Eucalyptus urophylla ST Blake in vitro. New Phytol 116, 115-122

Leyval C (1988) Interactions bactéries-mycorhizes dans la rhizosphère du pin sylvestre et du hêtre: incidences sur l'exsudation racinaire et l'altération des minéraux. Thèse doctorat ès Sciences, Univ Nancy I, Nancy 
Malajczuk N, Molina R, Trappe JM (1982) Ectomycorrhiza formation in Eucalyptus. 1. Pure culture synthesis, host specificity, and mycorrhizal compatibility with Pinus radiata. New Phytol 91, 467-482

Malajczuk N, Molina R, Trappe JM (1984) Ectomycorrhiza formation in Eucalyptus. II. The ultrastructure of compatible and incompatible mycorrhizal fungi and associated roots. New Phytol 96, 43-53

Malajczuk N, Lapeyrie F, Garbaye J (1990) Infectivity of pine and eucalypt isolates of Pisolithus tinctorius on roots of Eucalyptus urophylla in vitro. New Phytol 114, 627-631

Marx DH (1969) The influence of ectotrophic mycorrhizal fungi on the resistance of pine roots to pathogenic infection. I. Antagonism of mycorrhizal fungi to root pathogenic fungi and soil bacteria. Phytopathol 59, 153-163
Marx DH (1981) Variability in ectomycorrhizal development and growth among isolates of Pisolithus tinctorius as affected by source, age, and reisolation. Can J For Res 11, 168-174

Marx DH, Kenney DS (1982) Production of ectomycorrhizal fungus inoculum. In: Methods and Principles of Mycorrhizal Research (NC Schenk, ed) Am Phytopathol Soc, St Paul, MN, 131-146

Molina R, Trappe JM (1982) Patterns of ectomycorrhizal host specificy and potential among Pacific Northwest conifers and fungi. Forest Sci 28, 423-458

Thoen D, Ducousso M (1989) Mycorrhizal habit and sclerogenesis of Phlepobus sudanias (Gyrodontaceae) in Senegal. Agric Ecosystems and Environment 28, 519-523

Thoen D, Sougoufara B, Dommergues $Y(1990)$ In vitro mycorrhization of Casuarina and Allocasuarina species by Pisolithus tinctorius. Can $J$ Bot 68, 2537-2542 